\title{
Functional molecular electronic devices through environmental control
}

\author{
Dingkai $\mathrm{Su}^{\dagger}$, Chunhui $\mathrm{Gu}^{\dagger}$ and Xuefeng Guo
}

Motivated by Moore's law, molecular electronic devices are considered as a promising alternative of traditional silicon-based electronic devices due to their extreme miniaturization, low-cost manufacturing and versatility [1]. The initial model of molecular electronics was a molecular diode theoretically proposed by Aviram and Ratner in 1974 [2], where they utilized a single organic molecule to control the electric current flow in one-way direction. They designed an asymmetric molecule consisting of a donor- $\sigma$-acceptor structure $(\mathrm{D}-\sigma-\mathrm{A})$ to mimic a $\mathrm{p}-\mathrm{n}$ junction in semiconductors. At that time, one fundamental impediment that limited the realization of molecular diodes was how to immobilize a single molecule to a pair of electrodes. In terms of this issue, in the 1980s, the development of scanning tunneling microscopy (STM) and atomic force microscopy (AFM) made it feasible to probe the conductance of molecules. Until 1997, Reed et al. [3] developed the mechanically controllable break junction (MCBJ) technique to realize the measurement of an individual molecule and a real singlemolecule diode was fabricated later by this technique [4]. With the development of more and more approaches to build single-molecule junctions such as electromigration break junctions [5], STM break junctions [6] and carbon point contacts [7], researchers turned more attention to the functionalization of molecular electronic devices, the initial goal of molecular electronics. So far, molecular electronic devices have been successfully developed as prototypes with different functionalities including diodes [8], transistors [9], switches [10] and memories [11].

The realization of functionalities in molecular junctions is the synergistic effect of molecular engineering, interfacial engineering and materials engineering as shown in Fig. 1a. Firstly and most importantly, molecular engineering plays a key role in the device functionalization
$[12,13]$ because specially designed molecules are able to endow the intrinsic molecular properties, which are directly correlated to their electronic structures, into molecular electronic devices. Secondly and complementarily, interfacial engineering plays an important role in the electrical characteristics of molecular devices [14]. Through the design of different anchoring groups and/or the introduction of different spacers between the functional core and the anchoring group, interfacial engineering determines the electronic structure at the molecule-electrode contact interface, as well as the coupling strength between the molecules and the electrodes. As a result, the interfacial behavior effectively controls molecular conductance, charge transport mechanism and the performance of device functionalities. Finally and technically, materials engineering mainly involves the design and choice of electrode materials, thus determining the development of the device fabrication methodology and experimental methods. Currently, the widely applied electrode materials include metal $(\mathrm{Au}, \mathrm{Pt}, \mathrm{Cu}$, etc.), low-dimensional carbon materials (single-walled carbon nanotubes [15] and graphene [16]), and their combination [17]. Among these electrode materials, metallic electrodes benefit from easy repeatability and high throughput. However, they suffer from unstable contact, atom electromigration and stochastic transition of interface conformation. In contrast, carbon-based electrodes benefit from high stability, excellent size compatibility with molecules and robust interface conformations, overcoming the disadvantages of metal electrodes to some extent [18].

In general, the molecular orbitals (usually the highest occupied molecular orbital (HOMO) and the lowest unoccupied molecular orbital (LUMO)) of the central functional groups play an important role in determining

Beijing National Laboratory for Molecular Sciences, State Key Laboratory for Structural Chemistry of Unstable and Stable Species, College of Chemistry and Molecular Engineering, Peking University, Beijing 100871, China

These authors contributed equally to this paper.

*Corresponding author (email: guoxf@pku.edu.cn) 

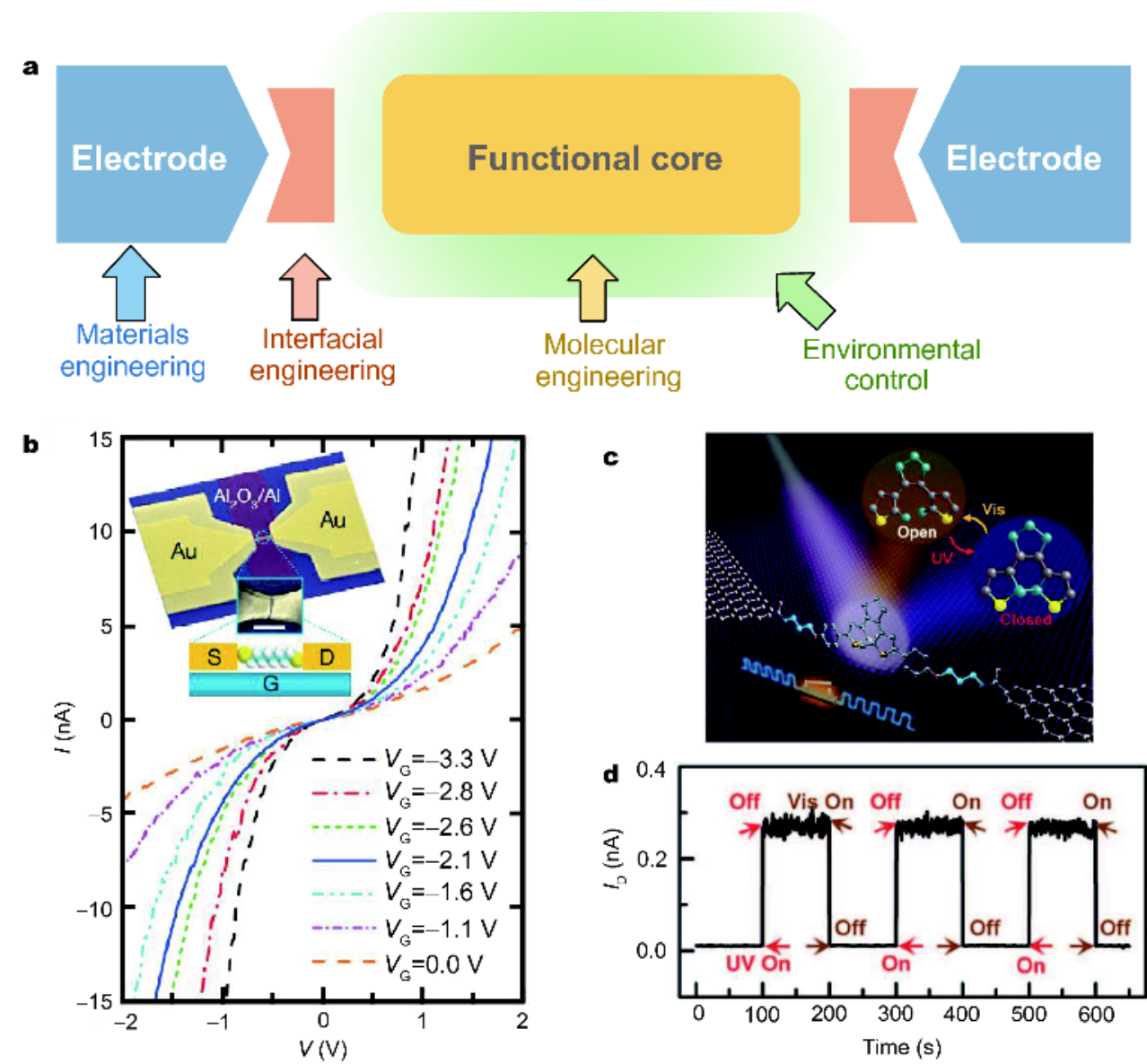

Figure 1 (a) Schematic of functional molecular electronic devices through environmental control. (b) Schematic of a single-molecule device and representative $I-V$ curves measured at $4.2 \mathrm{~K}$ for different values of $V_{\mathrm{G}}$. S, D and G represent source, drain and gate, respectively. (c) Schematic of a single-molecule transistor based on a graphene-diarylethene-graphene structure. (d) $I-t$ curve through a diarylethene molecule that reversibly switches between the closed and open forms, upon exposure to ultraviolet (UV) and visible (Vis) radiation, respectively. $V_{\mathrm{D}}=100 \mathrm{mV}$ and $V_{\mathrm{G}}=0 \mathrm{~V}$. Reproduced with permission from Ref. [9], Copyright 2009, Nature Publishing Group, and Ref. [24], Copyright 2016, American Association for the Advancement of Science.

the transport properties in molecular electronic devices because they provide spatial conduction channels for electron to transport and cover all the quantum mechanical information of the electronic structures. In addition to the intrinsic molecular structure, external manipulation of the molecular orbitals proves to be a direct and effective strategy to control the electron transport behavior. By taking advantage of the sensitivity of molecular orbitals to external stimuli such as an electric field $[19,20]$ and light $[21-23]$, the functionalities of molecular electronic devices can be largely enriched. For instance, in 2009, Song et al. [9] fabricated a threeterminal field-effect transistor (FET)-like molecular device. The device configuration in Fig. $1 \mathrm{~b}$ shows that individual molecules are bonded to source and drain electrodes $(\mathrm{Au})$ on the back gate electrode $\left(\mathrm{Al}_{2} \mathrm{O}_{3} / \mathrm{Al}\right)$.
The FET behavior demonstrated in this device is attributed to the pronounced effect of an external gate voltage in tuning the position of the HOMO or the LUMO relative to the Fermi level of the electrodes. In 2016, Jia et al. [24] realized a fully reversible single-molecule photoswitch via covalently sandwiching single diarylethenes between graphene point contacts (Fig. 1c). Through alternative radiation by ultraviolet and visible lights, the conformational change between closed/open forms of diarylethene molecules resulted in a smaller/ larger HOMO-LUMO gap, and thus led to a higher/lower conductance (Fig. 1d). These studies demonstrate that functional molecules can indeed be used as a functional component with diversity in constructing electrical nanocircuits.

It should be mentioned that the use of chemical stimuli 

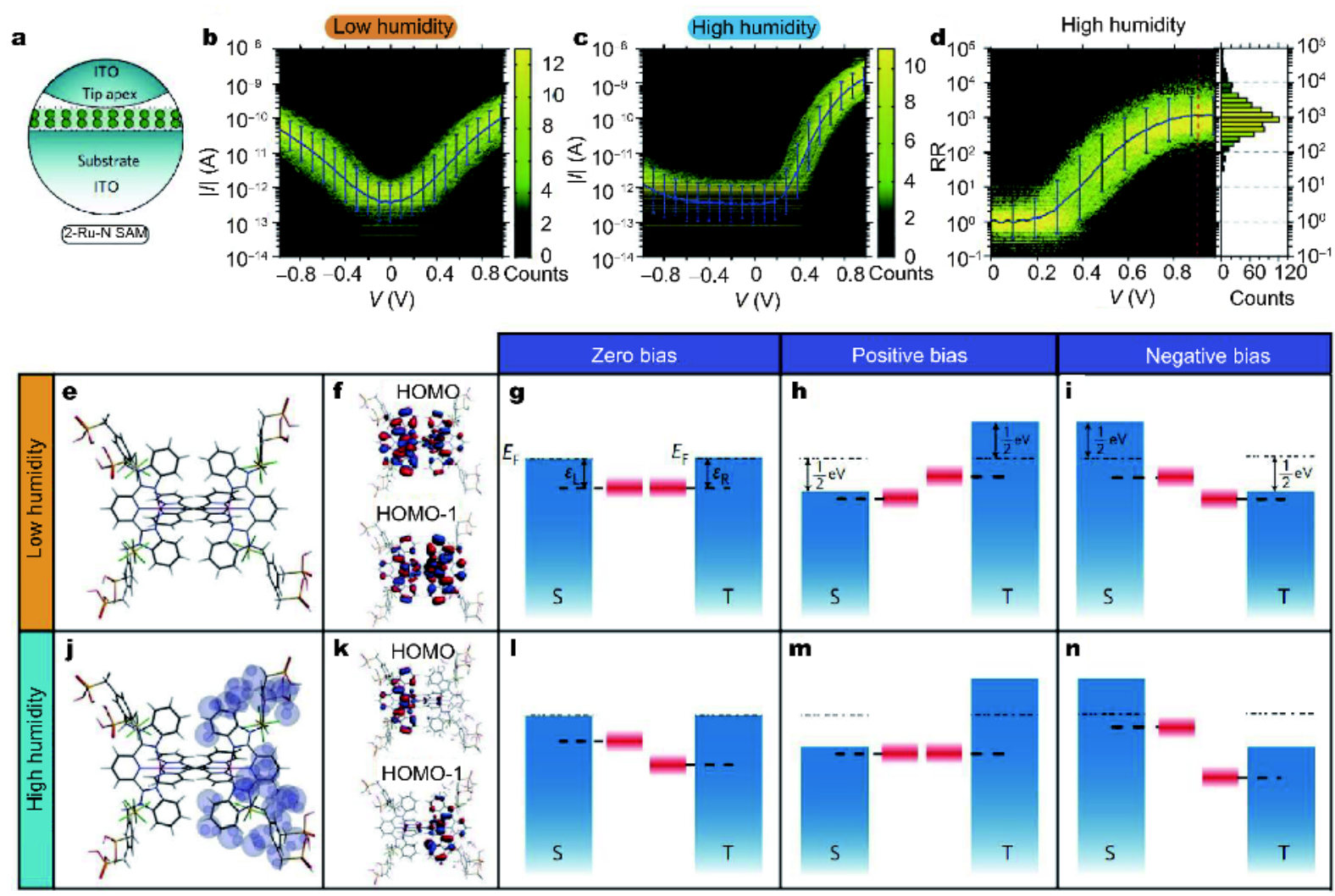

Figure 2 (a) Schematic of a 2-Ru-N ITO-molecule-ITO junction. (b, c) 2D histograms of logarithmically binned $I-V$ curves of 2-Ru-N molecular junctions for low humidity $(5 \%, \mathrm{~b})$ and high humidity $(60 \%, \mathrm{c})$ conditions. (d) Left, $2 \mathrm{D}$ histogram of logarithmically binned RR for the humid case. Right, $1 \mathrm{D}$ histogram taken at $V=0.9 \mathrm{~V}$. (e, j) The 2-Ru-N complex in simulated dry (e) and humid (j) conditions, with the purple dots representing water molecules. ( $f, k)$ The HOMO and HOMO-1 orbitals without (f) or with $(k)$ the existence of water. (g-i, l-n) Illustration of the two LMOs between substrate $(\mathrm{S})$ and tip $(\mathrm{T})$ electrodes at zero bias $(\mathrm{g}, \mathrm{l})$, positive bias $(\mathrm{h}, \mathrm{m})$ and negative bias $(\mathrm{i}, \mathrm{n})$ in dry and humid conditions, respectively. The difference between the Fermi energy and the LMOs is represented by $\varepsilon_{\mathrm{L}}$ (tip side) and $\varepsilon_{\mathrm{R}}$ (substrate side). Reproduced with permission from Ref. [25]. Copyright 2017, Nature Publishing Group.

to modulate the properties of molecular electronic devices has become a hot topic these years. In comparison with electrical field and light stimuli, chemical reactions on the molecular wires can directly and largely alter the electronic structure of molecular devices (and thus the corresponding device conductance). For example, recently, Atesci et al. [25] realized a novel humidity-controlled molecular diode based on a self-assembly monolayer (SAM) of di-nuclear Ru-complex (2-Ru-N) molecules by employing conductive-probe atomic force microscopy (C-AFM). The 2-Ru-N molecule has a symmetric backbone with a strong electrostatic coupling between the two $\mathrm{Ru}$-centers. The current-voltage $(I-V)$ characteristics were measured by trapping the SAM between two indium tin oxide (ITO) electrodes at low $(\sim 5 \%)$ or high $(\sim 60 \%)$ relative humidity (Fig. 2a). As shown in Fig. 2b, c, the $I-V$ characteristics of $2-\mathrm{Ru}-\mathrm{N}$ junctions in the dry condition are symmetric, whereas there is a clear asymmetry in the high-humidity curves. The fitted rectification ratio (RR) by a Gaussian curve at $0.9 \mathrm{~V}$ in high humidity condition $\left(\mathrm{RR}=10^{3.0 \pm 0.6}\right)$ is about three orders of magnitude higher than that in low humidity $\left(\mathrm{RR}=10^{0.4 \pm 0.4}\right)$ (Fig. 2d). Control experiments were conducted on mono-nuclear symmetric $\mathrm{Ru}$-complexes $1-\mathrm{Ru}-\mathrm{N}$ and asymmetric $\mathrm{Ru}$-complexes $1-\mathrm{Ru}-\mathrm{Py}$, but neither of them showed diode-like behaviors both in dry and humid conditions, which implied that the key factors to humidity-dependent rectification result from the interactions of two $\mathrm{Ru}$-centers in series and water molecules. Quantum chemistry calculations explained the mechanism of humidity-dependent rectification in $2-\mathrm{Ru}-\mathrm{N}$ junctions. In the dry circumstances, the two localized molecular orbitals (LMOs), which is the combination of HOMO and HOMO-1, are degenerate between the tip and the substrate (Fig. 2f, g). Under positive or negative biases, there is a symmetric decrease in transmission as the two levels shift with each other (Fig. 

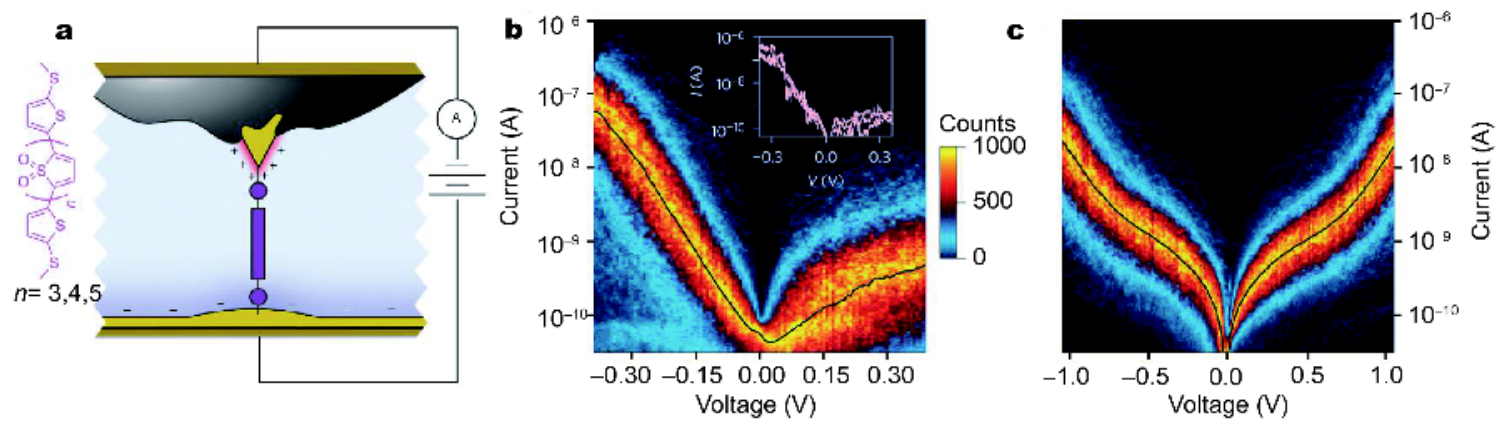

d

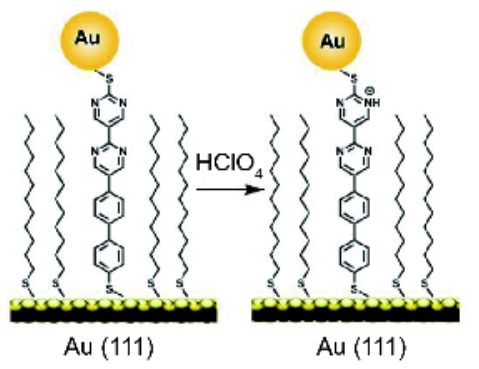

$\mathbf{f}$

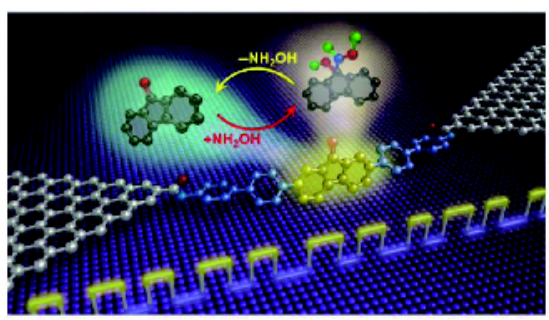

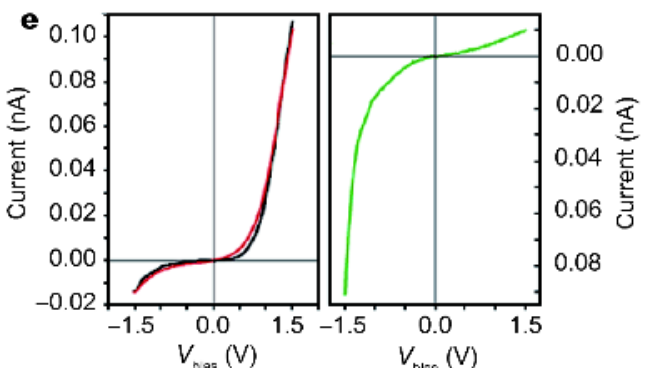

$\mathbf{g}$

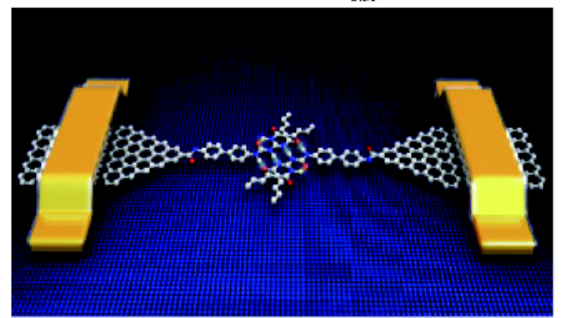

Figure 3 (a) TDOn molecular structure and schematic of a molecular junction with asymmetric area electrodes. (b, c) 2D histograms of logarithmically binned $I-V$ curves of TDO4 in PC (b) and TCB (c). Inset in (b): examples of exceptionally rectifying junctions. (d) Self-assembly of diblock molecular diodes and protonation by $\mathrm{HClO}_{4}$. (e) $I-V$ curves of diblock before protonation (black), after protonation (green), and after protonation-deprotonation (red). (e) Schematic of a graphene-fluorenone-graphene junction. (f) Schematic of a graphene-quadruple hydrogen bond dimer-graphene junction. Reproduced with permission from Ref. [27], Copyright 2015, Nature Publishing Group, Ref. [29], Copyright 2016, American Chemical Society, Ref. [31], Copyright 2018, American Association for the Advancement of Science, and Ref. [32], Copyright 2018, Nature Publishing Group.

$2 \mathrm{~h}, \mathrm{i})$. In the humid circumstances, however, the two LMOs become misaligned, the chemical potential of the LMO near the tip was lower than that of the other LMO (Fig. 21). The reason for the level misalignment is that the replacement of the counter ions by water molecules at the tip side due to the higher hydrophilicity of the bare ITO tip and capillary effects breaks the asymmetry in the electrostatic environment of the tip and the substrate (Fig. $2 \mathrm{j}, \mathrm{k}$ ). This level misalignment induced asymmetric factors influencing the LMO gaps on the contrary when the bias was applied in opposite directions. The transport is enhanced as the gap between the two chemical potentials of LMOs decreases at the positive bias (Fig. $2 \mathrm{~m}$ ), while the transport is suppressed when the levels shift away from the resonance at the negative bias (Fig. 2n). In addition, researchers found that the junction was also responsive to tip radius and applied force in humidity. After the opti- mization of tip radius and background noise, $R R$ values could exceed 6,500.

Besides directly tuning the molecular orbitals, external chemical stimuli also take effect by controlling the electronic structure of the interface [26]. For instance, Capozzi et al. [27] used the STM break junction to characterize the electrical property of a symmetric oligomer of thiophene-1,1-dioxide (TDO) in polar solvents or nonpolar solvents, such as propylene carbonate (PC) or 1,2,4-trichlorobenzene (TCB) (Fig. 3a). Fig. 3b demonstrates that the molecule can rectify in $\mathrm{PC}$ and the fitted RR value is more than 200 at $0.37 \mathrm{~V}$, the highest reported for single-molecule diodes so far. By contrast, rectification behavior is not observed in TCB, as shown in Fig. 3c. The experiment and theoretical model reveal that the electric double layer formed at the tip is denser than that at the substrate in a polar and ion-soluble solvent 
because of the smaller area of the tip. Therefore, the molecular orbitals are pinned to the chemical potential of the substrate and the current depends on the polarity of the applied voltage.

Another result caused by chemical environment, which cannot be ignored in molecular electronic devices, is the charge distribution effect. It was reported by Morales et al. [28] that the rectification direction of single-molecule diodes can be switched responding to the acidity of the environment (Fig. 3d). The dipyrimidinyl-diphenyl diblock oligomer connected between the Au substrate and $\mathrm{Au}$ nanoparticle exhibits an evident rectification effect owing to its large dipolar moment (Fig. 3e, black line). With the addition of perchloric acid $\left(\mathrm{HClO}_{4}\right)$, the nitrogen atom on the pyrimidinyl moiety is protonated and the dipolar moment inverted, which results in the inversion of rectification direction (Fig. 3e, green line). After the deprotonation by sodium ethoxide, rectification direction could recover (Fig. 3e, red line) [29].

As discussed above, molecular electronic devices based on both single molecules and SAMs can function as rectifiers and be controlled through external environmental stimuli. In comparison with SAM-based devices, the rectification ratio and the sensitivity for single-molecule devices are less pronounced due to inevitable tunneling current at the "off" state. However, the advantages of single-molecule devices are their small size and their ability to reflect the unique behaviors of individual molecules. Notably, a special technique that combines chemical stimuli with high time-resolution acquisition was developed to monitor single-molecule junctions [30]. By real-time measurement, the dynamic process of chemical reactions can be revealed at the single-molecule/single-event level. This is a promising direction for future investigation in this field because the study of chemical reaction kinetics not only reveals the intrinsic mechanism of chemical reactions, but also can effectively regulate the chemical reaction, which is of practical significance to synthesis and chemical production. Recently, Guan et al. [31] developed a reliable platform of graphene-molecule-graphene single-molecule junctions to carry out single-molecule electrical detection in a solution environment with a time resolution of microseconds. With the combination of real-time electrical signals and theoretical calculation, the intermediate in a nucleophilic addition reaction of hydroxylamine to a carbonyl group was discovered (Fig. 3f). Furthermore, through direct observation of individual hydrogen bond dynamics in different solvents/temperatures, Zhou et al. [32] did observe fast stochastic rearrangements of the hydrogen bond structures mainly through intermolecular proton transfer and lactam-lactim tautomerism (Fig. 3g)

On the basis of the recent representative advances, we summarized and highlighted such a burgeoning area of environment-controlled molecular electronic devices. Starting from the structure-function relationship, functionalized molecular electronic devices are designed to be sensitive to specified external stimuli through molecular, interfacial and/or materials engineering. Essentially, the functionality of this type of molecular electronic devices is achieved through the modulation of molecular orbitals controlled by environmental stimuli, involving electric/ magnet field, light or chemicals. In particular, chemical stimuli have been developed rapidly as a powerful tool to control the behavior of molecular electronic devices with great diversity, selectivity and feasibility. This strategy provides a unique perspective for integrating numerous functionalities into electrical nanocircuits, which will invite intense studies in the future.

\section{Received 27 March 2018; accepted 14 May 2018;}

published online 22 June 2018

1 Xiang D, Wang X, Jia C, et al. Molecular-scale electronics: from concept to function. Chem Rev, 2016, 116: 4318-4440

2 Aviram A, Ratner MA. Molecular rectifiers. Chem Phys Lett, 1974, 29: $277-283$

3 Reed MA. Conductance of a molecular junction. Science, 1997, 278: $252-254$

4 Elbing M, Ochs R, Koentopp M, et al. A single-molecule diode. Proc Natl Acad Sci USA, 2005, 102: 8815-8820

5 Park J, Pasupathy AN, Goldsmith JI, et al. Coulomb blockade and the Kondo effect in single-atom transistors. Nature, 2002, 417: $722-725$

$6 \mathrm{Xu} \mathrm{B}$, Tao NJ. Measurement of single-molecule resistance by repeated formation of molecular junctions. Science, 2003, 301: 12211223

7 Cao Y, Dong S, Liu S, et al. Building high-throughput molecular junctions using indented graphene point contacts. Angew Chem Int Ed, 2012, 51: 12228-12232

8 Díez-Pérez I, Hihath J, Lee Y, et al. Rectification and stability of a single molecular diode with controlled orientation. Nat Chem, 2009, 1: 635-641

9 Song H, Kim Y, Jang YH, et al. Observation of molecular orbital gating. Nature, 2009, 462: 1039-1043

10 Su TA, Li H, Steigerwald ML, et al. Stereoelectronic switching in single-molecule junctions. Nat Chem, 2015, 7: 215-220

11 Green JE, Wook Choi J, Boukai A, et al. A 160-kilobit molecular electronic memory patterned at 1011 bits per square centimetre. Nature, 2007, 445: 414-417

12 Guo C, Wang K, Zerah-Harush E, et al. Molecular rectifier composed of DNA with high rectification ratio enabled by intercalation. Nat Chem, 2016, 8: 484-490

13 Ismael AK, Wang K, Vezzoli A, et al. Side-group-mediated mechanical conductance switching in molecular junctions. Angew Chem Int Ed, 2017, 56: 15378-15382 
14 Jia C, Guo X. Molecule-electrode interfaces in molecular electronic devices. Chem Soc Rev, 2013, 42: 5642-5660

15 Guo X, Small JP, Klare JE, et al. Covalently bridging gaps in singlewalled carbon nanotubes with conducting molecules. Science, 2006, 311: 356-359

$16 \mathrm{Gu}$ C, Su D, Jia C, et al. Building nanogapped graphene electrode arrays by electroburning. RSC Adv, 2018, 8: 6814-6819

17 Kim T, Liu ZF, Lee C, et al. Charge transport and rectification in molecular junctions formed with carbon-based electrodes. Proc Natl Acad Sci USA, 2014, 111: 10928-10932

18 Jia C, Ma B, Xin N, et al. Carbon electrode-molecule junctions: a reliable platform for molecular electronics. Acc Chem Res, 2015, 48: 2565-2575

19 Kim WY, Kim KS. Tuning molecular orbitals in molecular electronics and spintronics. Acc Chem Res, 2010, 43: 111-120

20 Wen J, Tian Z, Ma J. Light- and electric-field-induced switching of thiolated azobenzene self-assembled monolayer. J Phys Chem C, 2013, 117: 19934-19944

21 Pourhossein P, Vijayaraghavan RK, Meskers SCJ, et al. Optical modulation of nano-gap tunnelling junctions comprising self-assembled monolayers of hemicyanine dyes. Nat Commun, 2016, 7: 11749

22 Fung ED, Adak O, Lovat G, et al. Too hot for photon-assisted transport: hot-electrons dominate conductance enhancement in illuminated single-molecule junctions. Nano Lett, 2017, 17: 12551261

23 Zhou J, Wang $\mathrm{K}, \mathrm{Xu} \mathrm{B}$, et al. Photoconductance from exciton binding in molecular junctions. J Am Chem Soc, 2018, 140: 70-73

24 Jia C, Migliore A, Xin N, et al. Covalently bonded single-molecule junctions with stable and reversible photoswitched conductivity. Science, 2016, 352: 1443-1445

25 Atesci H, Kaliginedi V, Celis Gil JA, et al. Humidity-controlled rectification switching in ruthenium-complex molecular junctions.
Nat Nanotechnol, 2018, 13: 117-121

26 Barber JR, Yoon HJ, Bowers CM, et al. Influence of environment on the measurement of rates of charge transport across $\mathrm{Ag}^{\mathrm{TS}}$ / $\mathrm{SAM} / / \mathrm{Ga}_{2} \mathrm{O}_{3} / \mathrm{EGaIn}$ Junctions. Chem Mater, 2014, 26: 3938-3947

27 Capozzi B, Xia J, Adak O, et al. Single-molecule diodes with high rectification ratios through environmental control. Nat Nanotechnol, 2015, 10: 522-527

28 Morales GM, Jiang P, Yuan S, et al. Inversion of the rectifying effect in diblock molecular diodes by protonation. J Am Chem Soc, 2005, 127: 10456-10457

29 Lo WY, Zhang N, Cai Z, et al. Beyond molecular wires: design molecular electronic functions based on dipolar effect. Acc Chem Res, 2016, 49: 1852-1863

30 Gu C, Jia C, Guo X. Single-molecule electrical detection with realtime label-free capability and ultrasensitivity. Small Methods, 2017, 1: 1700071

31 Guan J, Jia C, Li Y, et al. Direct single-molecule dynamic detection of chemical reactions. Sci Adv, 2018, 4: eaar2177

32 Zhou C, Li X, Gong Z, et al. Direct observation of single-molecule hydrogen-bond dynamics with single-bond resolution. Nat Commun, 2018, 9: 807

Acknowledgements We acknowledge primary financial supports from the National Key R\&D Program of China (2017YFA0204901), the National Natural Science Foundation of China (21727806) and the Natural Science Foundation of Beijing (Z181100004418003).

Author contributions Guo X conceived the structure of the manuscript and $\mathrm{Su} \mathrm{D}$ and $\mathrm{Gu} \mathrm{C}$ wrote the initial paper. All the authors discussed the results and commented on the manuscript. Su D and Gu C contributed equally to the work.

Conflict of interest The authors declare no conflict of interest. 

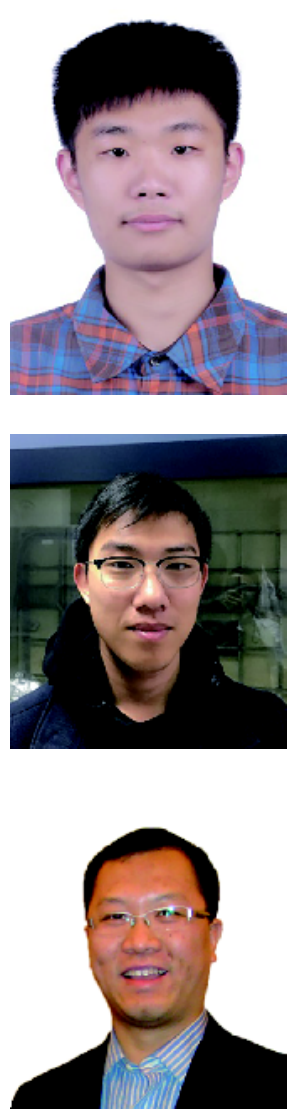

Dingkai Su received his BSc degree in 2017 from the College of Nano Science and Technology, Soochow University. He is currently a $\mathrm{PhD}$ candidate at the College of Chemistry and Molecular Engineering, Peking University, under the supervision of Prof. Xuefeng Guo. His research interest focuses on the single-molecule devices and dynamics.

Chunhui Gu received his BSc degree in 2014 from the College of Chemistry and Molecular Engineering, Peking University. He is a PhD candidate at the College of Chemistry and Molecular Engineering, Peking University, under the guidance of Prof. Xuefeng Guo. His current research interest includes single-molecule devices and dynamics.

Xuefeng Guo received his PhD degree in 2004 from the Institute of Chemistry, CAS, Beijing. From 2004 to 2007, he was a postdoctoral research scientist at Columbia University Nanocenter. He joined the faculty as a professor at Peking University in 2008. His current research focuses on functional nanometer/molecular devices.

\section{环境调控的功能分子电子器件}

苏鼎凯 ${ }^{\dagger}$, 顾春晖 ${ }^{\dagger}$, 郭雪峰 ${ }^{*}$

摘要 为了满足传统硅基电子器件日益微型化的需求, 科学家们前瞻性地提出了将单个分子或者分子聚集体夹在电极之间制备分子电子 器件的前沿研究方向. 分子电子器件功能化的实现需要从分子工程、界面工程以及材料工程的角度综合考虑. 本文总结了近期利用外界 环境来调控分子电子器件功能的最新进展. 鉴于化学刺激所引起的显著效果, 作者展望了分子电子器件在单分子化学反应动力学中的应 用前景以及未来的发展方向. 